\title{
Análise, Desenvolvimento e Avaliação de um Objeto de Aprendizagem para Ensino Colaborativo de Frações, Números Decimais e Porcentagem
}

\author{
Irmgard A. H. C. Nakazoni ${ }^{1}$, Thiago M. Alexandrino ${ }^{2}$, Ulises J. A. Garrido ${ }^{2}$, \\ Isabela Gasparini $^{1,2}$, Elisa Henning ${ }^{2}$ \\ ${ }^{1}$ PPGCA e ${ }^{2}$ PPGECMT - Universidade do Estado de Santa Catarina (UDESC) \\ irmgard.nakazoni@hotmail.com, thiago.alexandrinodedu.sesisc.org.br, \\ ulises.aguayogarrido@gmail.com, isabela.gasparini@udesc.br, \\ elisa.henning@udesc.br
}

Resumo. Este trabalho tem por finalidade apresentar as etapas do Design Instrucional (DI) de um Objeto de Aprendizagem (OA) em que o objetivo principal é a exploração e aprendizagem colaborativa de conteúdos matemáticos específicos (Frações, Números Decimais e Porcentagem). Considerando a metodologia ADDIE para o DI, este artigo aborda as etapas de Análise, Design, Desenvolvimento, Implementação com ênfase na etapa de Avaliação. Os resultados obtidos com o estudo de caso realizado na etapa de Avaliação, ratificam a importância da adoção de uma metodologia para o DI de OAs, especialmente no que se refere à interdisciplinaridade destas soluções.

Palavras-chave: Ensino de Matemática, Design Instrucional, ADDIE.

\section{Analysis, Development and Evaluation of a Learning Object to Collaborative Learning of Fractions, Decimal Numbers and Percentage}

\begin{abstract}
This work aims to present the Instructional Design (ID) stages of a Learning Object (LO) in which the main goal is the exploration and collaborative learning of specific mathematical content (Fractions, decimal numbers and percentage). Considering the ADDIE methodology for DI, this paper discusses the Analysis, Design, Development, Implementation, and emphasizing the Evaluation stage. The results obtained from the case study in the evaluation stage confirm the importance of adopting a methodology for the ID of LOs, especially with regard to interdisciplinarity of these solutions.
\end{abstract}

Keywords: Mathematics Teaching, Instructional Design, ADDIE.

\section{Introdução}

Entende-se como Objetos de Aprendizagem (OA) todo e qualquer recurso digital que possa ser usado, reutilizado ou referenciado durante a aprendizagem apoiada por tecnologia (WILEY, 2002, p. 115-126). Do ponto de vista tecnológico, eles são objetos autocontidos, marcados por descritores de identificação que, também denominados metadados, podem ser pesquisados e compartilhados em ações educacionais (FILATRO, 2008, p. 123). A implementação de objetos de aprendizagem tem entre seus pré-requisitos a utilização de uma metodologia que se proponha a suportar todas as etapas do processo de forma que o resultado final se converta na aprendizagem dos conteúdos propostos por meio de sua utilização. 
A aprendizagem colaborativa pode ser entendida como a troca de saberes e tem sido defendida por educadores e praticada por muitos professores nos diversos níveis escolares. Apesar desta prática não ser uma novidade, para Castro (2011) a disponibilidade das tecnologias de comunicação e de interação social tem contribuído para melhorias e a adesão de novos interessados. Entre os benefícios desta prática pedagógica, destacam-se: a preparação para a vida em sociedade, o desenvolvimento do espírito crítico e a competência para resolver problemas de grande porte a partir das contribuições individuais.

O objeto de aprendizagem implementado tem o intuito de trabalhar conteúdos matemáticos específicos em um Ambiente Virtual de Aprendizagem (AVA) de forma colaborativa. A metodologia utilizada para o Design Instrucional de Objetos de Aprendizagem apresentada nesse artigo foi o Modelo ADDIE (Analysis, Design, Development, Implementation, Evaluation - em Português Análise, Projeto, Desenvolvimento, Implementação, Avaliação). Suas etapas serão descritas em cada uma das seções desse artigo: Análise, Projeto, Desenvolvimento, Implementação com ênfase na seção de Avaliação. Na sequência, serão apresentados e discutidos os resultados obtidos na etapa de Avaliação e por fim, serão apresentadas as considerações finais referentes ao processo de DI dessa solução.

\section{Análise (Analysis)}

A metodologia ADDIE, de acordo com Filatro (2008), considera que a etapa de análise consiste na compreensão do problema educacional bem como na projeção de sua solução aproximada. Na sequência, apresentam-se os levantamentos das necessidades educacionais, a caracterização do público-alvo, objetivos e restrições.

Desde sua concepção, o OA implementado teve como premissa a "Aprendizagem Colaborativa de Conteúdos Matemáticos" sendo disponibilizado para utilização on-line de forma colaborativa àqueles interessados em seu conteúdo através da ferramenta AdaptWeb ${ }^{\circledR}$ (Ambiente de Ensino-aprendizagem Adaptativo na Web) que é um sistema hipermídia adaptativo de educação a distância baseado na Web que tem como finalidade adaptar o conteúdo, a apresentação e a navegação de acordo com o modelo do usuário (GASPARINI, 2003).

Os conteúdos foram disponibilizados na ferramenta AdaptWeb ${ }^{\circledR}$ e organizados em tópicos, sendo que cada tópico abordado foi dividido de acordo com a estrutura do Ambiente, em: Conceito, Exemplos, Exercícios e Material Complementar e a interação entre os estudantes pode ser realizada via Fórum de Discussão, Mural de Recados e Chats $^{l}$. As avaliações de aprendizagem podem ser colaborativas e individuais para cada tópico abordado.

No que tange ao público alvo, o OA pretende atender aos conteúdos curriculares específicos de estudantes do $5^{\circ}$ Ano do Ensino Fundamental I, podendo estender-se a todo aquele que tenha interesse por seu conteúdo e que tenha apropriado os requisitos mínimos para compreensão dos mesmos. Salienta-se ainda que a proposta favorece àqueles que se interessem pela forma colaborativa de aprendizagem. 
Do ponto de vista pedagógico, os seguintes objetivos devem ser atingidos mediante a utilização do OA implementado: (a) interpretar diferentes tipos de desenhos e gráficos referentes a frações e porcentagens; (b) aprender a resolver exercícios e problemas de frações e porcentagens; (c) conhecer e diferenciar diferentes tipos de problemas de frações e porcentagens; e (d) aprender sobre as características de diferentes tipos de problemas de frações e porcentagens.

Considerando o caráter multidisciplinar do OA, a equipe de trabalho foi constituída desde a etapa de análise até a de avalição pelos seguintes especialistas: (a) um profissional da área de Tecnologia da Informação; e (b) dois docentes com formação em Matemática.

A abordagem pedagógica considerada na concepção do OA foi a sóciointeracionista de Vygostky que privilegia as relações sociais, o uso de instrumentos e a mediação como base do conhecimento que, de acordo com Vygotsky, é socialmente construído e culturalmente transmitido. Essa abordagem entende que a aprendizagem ocorre cada vez que a "Zona de Desenvolvimento Potencial" se transforma em "Zona de Desenvolvimento Real" por conta da ação do mediador na "Zona de Desenvolvimento Proximal". Esta mediação é realizada por meio de instrumentos e de um sistema simbólico. Assim sendo, foi possível estabelecer as seguintes relações:

- Zona de Desenvolvimento Real: conhecimentos prévios que o usuário do OA possui e, neste contexto, são considerados mínimos para compreensão e aprendizagem dos conceitos matemáticos propostos;

- Zona de Desenvolvimento Potencial: conhecimento a ser adquirido dos conceitos de Frações, Números Decimais e Porcentagem;

- Zona de Desenvolvimento Proximal: é o "delta" entre a Zona de Desenvolvimento Proximal e a Zona de Desenvolvimento Real. É nesta área em que o mediador irá atuar para a promoção da aprendizagem. Vale ressaltar que, neste contexto, não há somente um mediador, mas sim, esta função poderá ser exercida pelo usuário do OA que tiver um conhecimento maior do que os demais envolvidos no assunto/conceito abordado;

- Sistema Simbólico: refere-se a "linguagem" utilizada para transmissão do conhecimento, por exemplo, os signos utilizados nos conceitos matemáticos propostos e o vídeo tutorial.

- Instrumento: representado pelo OA implementado.

Filatro (2008) ratifica que tal abordagem, também denominada "Construtivista Social", implica ambientes colaborativos com desafios apropriados, encorajamento à experimentação e à descoberta compartilhadas, foco em conceitos e habilidades existentes, além da modelagem de habilidades, inclusive sociais.

Quanto ao modelo de estilo de aprendizagem o OA baseia-se no modelo proposto por Felder e Silverman (1998) e atualizado por Felder e Brent (2005). Considerando as propriedades da mesma, acredita-se que sejam favorecidos os aprendizes que, privilegiem a aprendizagem conforme as dimensões abaixo (SANTOS; MONGON, 2010):

- ativos: tendem a compreender melhor as informações, discutindo, aplicando ou explicando para os outros; 
- intuitivos: frequentemente saem-se melhor com conceitos, abstrações e fórmulas matemáticas; gostam de inovação e não apreciam a repetição, são rápidos e criativos;

- visuais: lembram-se mais facilmente de figuras, diagramas e de filmes;

- sequenciais: avançam com entendimento parcial, preferem a lógica e aprendem melhor por meio de conteúdos lineares, com cada passo derivando do anterior.

\section{Projeto (Design)}

Esta etapa contempla o planejamento e o design da situação didática propriamente dita (FILATRO, 2008). As informações foram expressas por meio das Unidades de Aprendizagem, Matriz de DI e Projeto dos Componentes. Conforme Filatro (2008), Unidade de Aprendizagem (UA) é uma unidade atômica ou elementar que contém os elementos necessários ao processo de ensino-aprendizagem e sua granularidade é definida pelos seguintes aspectos:

- uma Unidade de Aprendizagem não pode ser subdividida em partes sem perder o significado;

- tem extensão e tempo limitados; e

- é autocontida no que se refere a processos, objetivos e conteúdos.

As unidades de aprendizagem deste $\mathrm{OA}$ foram didaticamente agrupadas em macro-unidades com a finalidade de relacionar conteúdos afins, determinando assim uma relação de interdependência e sequenciamento conforme apresentado na Figura 1.

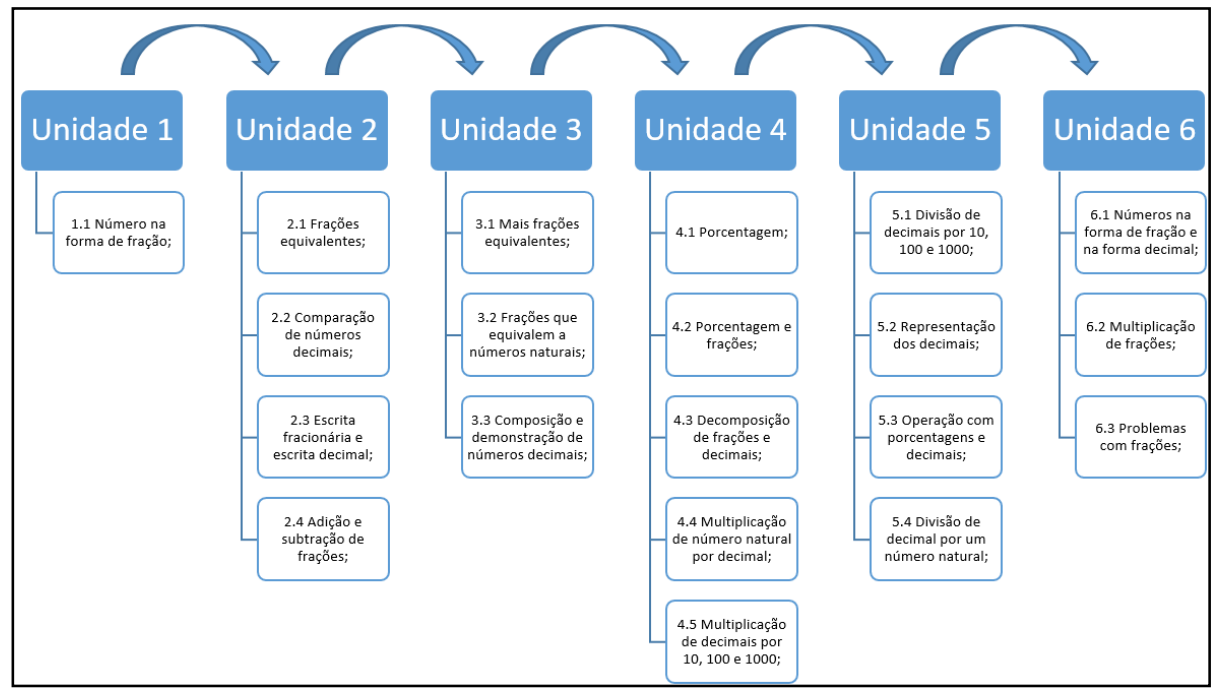

Figura 1 - Unidades de Aprendizagem e Estrutura Organizacional

A matriz de design instrucional (MDI) proporciona uma visão panorâmica de cada unidade de aprendizagem, explicitando quais atividades serão necessárias ao atendimento dos objetivos propostos (FILATRO, 2008). Neste trabalho, para cada unidade de aprendizagem, a MDI contém: objetivo da unidade, conteúdo a ser abordado, cursos vinculados, pré-requisitos necessários, qual a forma de entrega e como é avaliado.

Em relação ao projeto dos componentes, as unidades de aprendizagem contempladas foram disponibilizadas no AdaptWeb ${ }^{\circledR}$ sendo que a utilização desta 
ferramenta, enquanto ambiente virtual de aprendizagem, precede de sua configuração para contemplar os conteúdos desejados. Com base na M DI, Estrutura Organizacional e dos materiais elaborados pela equipe de docentes, os conteúdos foram atualizados no ambiente pelo profissional de tecnologia da informação.

\section{Desenvolvimento (Development)}

Considera-se esta etapa, como sendo a produção e a adaptação dos recursos e materiais didáticos impressos e/ou digitais, a parametrização de ambiente virtuais e a preparação dos suportes pedagógico, tecnológico e administrativo (FILATRO, 2008). Sendo uma etapa de cunho prático e executivo, seus resultados serão explicitados na seção a seguir sobre a Implementação.

\section{Implementação (Implementation)}

De acordo com o Filatro (2008), a implementação constitui a situação didática propriamente dita, quando ocorre a aplicação da proposta de design instrucional e subdivide-se aqui em duas fases: publicação e execução.

O projeto, em sua totalidade, foi configurado para ofertar seis unidades de aprendizagem e estas foram subdividas em tópicos específicos. Todas as Unidades apresentadas na Figura 1 foram implantadas no AdaptWeb ${ }^{\circledR}$, em relação aos respectivos conceitos abordados (tópicos explorados e explicação teórica sobre o tema). Entretanto, somente a Unidade 1 foi desenvolvida na íntegra, ou seja, além dos conceitos, também foram preparados exemplos, exercícios, outros materiais complementares e uma avaliação.

\section{Avaliação (Evaluation)}

Esta etapa consiste nas considerações sobre a efetividade da solução proposta bem como a revisão das estratégias implementadas (FILATRO, 2008). Optou-se pela realização de uma avaliação na forma de um estudo de caso e de cunho qualitativo em que a utilização da ferramenta foi realizada por duas alunas do $5^{\circ}$ Ano de uma Escola Fundamental I, com idades entre 11 e 12 anos. Para tal, elas foram convidadas a irem a um laboratório na Universidade do Estado de Santa Catarina (UDESC). Inicialmente, todas as orientações foram repassadas aos participantes, explicando os objetivos do estudo aos estudantes e seus responsáveis. Os responsáveis assinaram um Termo de Consentimento Livre Esclarecido, autorizando a participação de suas dependentes bem como a utilização dos resultados obtidos no processo avaliativo.

Os seguintes instrumentos foram utilizados para avaliação do OA: um Questionário chamado Inventário de Estilo de Aprendizagem (ILS) de Felder adaptado para o idioma português (ILSQ, 2015), Desempenho nos Exercícios (Colaborativo), Desempenho nas Avaliações (Individual), Informações disponíveis na Ferramenta de Análises de Aprendizagem (extraídas do AdaptWeb), Questionário de Satisfação e Observações (Ensaio de Interação). A apuração dos resultados foi efetuada por meio da triangulação dos resultados obtidos nos instrumentos de avaliação. 


\section{Apresentação dos Resultados}

Os resultados apresentados a seguir referem-se a etapa de avaliação realizada em Novembro de 2015. A avaliação contou com a participação de duas estudantes do sexo feminino denominadas de Participante 01 e Participante 02. A Participante 01 tem 11 anos e cursa o $5^{\circ}$ Ano do Ensino Fundamental I em uma instituição particular enquanto a Participante 02 tem 12 anos e cursa o $6^{\circ}$ Ano do Ensino Fundamental II em uma instituição pública. Ambas se conheciam previamente à realização da avaliação. A avaliação seguiu o protocolo de aplicação desenvolvido pela equipe do projeto. A seguir, o detalhamento dos resultados obtidos na etapa de avaliação.

\subsection{Resultados do Inventário de Estilo de Aprendizagem (ILS)}

Nesta seção é apresentado o resultado do ILS de cada uma das participantes. Estas informações foram obtidas utilizando a correção automática disponível em ILSQ (2015). O ILS organiza o Estilo de Aprendizagem em quatro dimensões propostas por Felder e Silverman (1998) e adaptadas por Felder e Brent (2005): (1) Ativo x Reflexivo, (2) Sensorial x Intuitivo, (3) Visual x Verbal e (4) Sequencial x Global. O resultado é apresentado para cada dimensão. Quando o resultado é apresentado na escala de 1-3, isso significa a faixa de equilíbrio, não havendo preferência a um determinado estilo; quando o resultado está em 5-7, indica preferência modera, (i.e. o estudante aprende com mais facilidade em um ambiente de ensino que favoreça o estilo apresentado), e quando o resultado está em 9 - 11, há uma forte preferência a um determinado estilo, e o estudante pode apresentar dificuldade caso o ambiente desconsidere este estilo.

As participantes preencheram o ILS sob a orientação de um dos integrantes da equipe e posteriormente suas respostas foram submetidas à correção on-line apresentando os resultados, que podem ser visualizados na Figura 2.

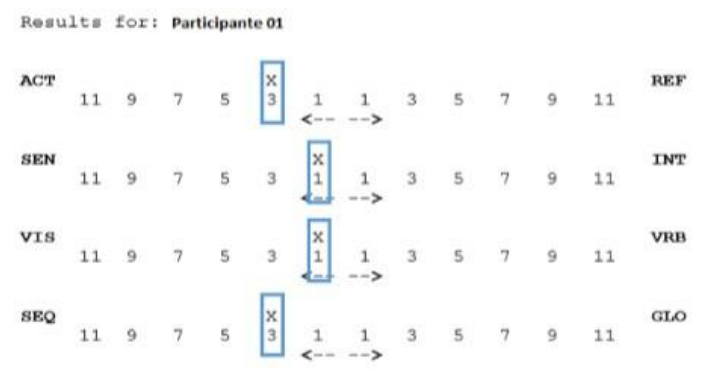

(a)

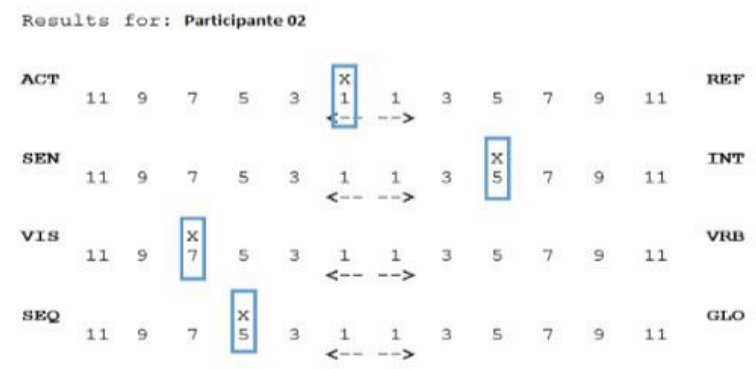

(b)

Figura 2 - (a) ILS Participante 01 e (b) ILS Participante 02

Analisando a Figura 2, pode-se constatar que a Participante 01 tem uma preferência equilibrada entre as dimensões. Isso expressa que ela pode receber diferentes tipos de materiais, adequados aos diferentes estilos, sem afetar seu processo de aprendizagem. Já a Participante 02 possui uma preferência equilibrada entre os estilos Ativo e Reflexivo e preferências moderadas para os estilos Intuitivo, Visual e Sequencial, ou seja, o ambiente deve estar preparado, com materiais adequados a estes estilos. Vale ressaltar que tanto os materiais elaborados quando o próprio ambiente AdaptWeb ${ }^{\circledR}$ foram preparados de modo a atender os diferentes estilos de aprendizagem. 


\subsection{Progresso e Desempenho Geral}

As informações a seguir foram extraídas da ferramenta de Análise de Aprendizagem do AdaptWeb $^{\circledR}$ e do score obtido na realização dos exercícios realizado de forma colaborativa e da avaliação final realizada individualmente. As Figuras 3(a) e 3(b) apresentam respectivamente um OA desenvolvido e o menu navegacional por todas as unidades estudadas. Em relação às cores apresentadas no menu da Figura 3(b), os links estão em azul representam conceitos ainda não visitados, os links na cor púrpura representam conceitos já acessados e o link na cor vermelha representa o conceito que está sendo estudado no momento.

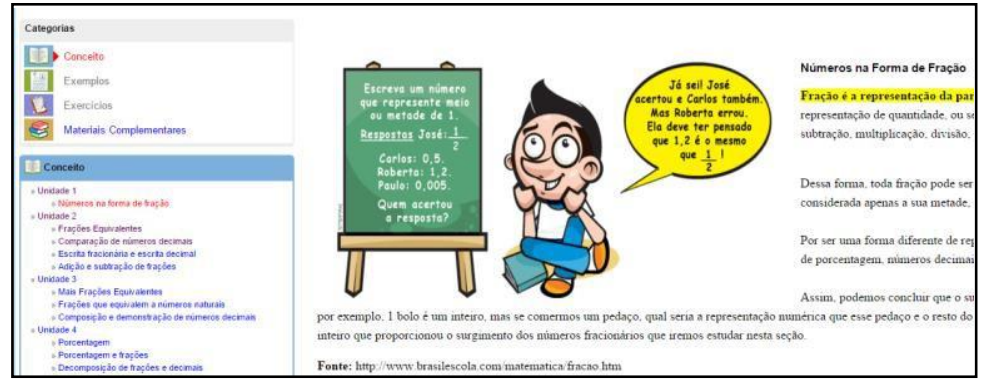

(a)

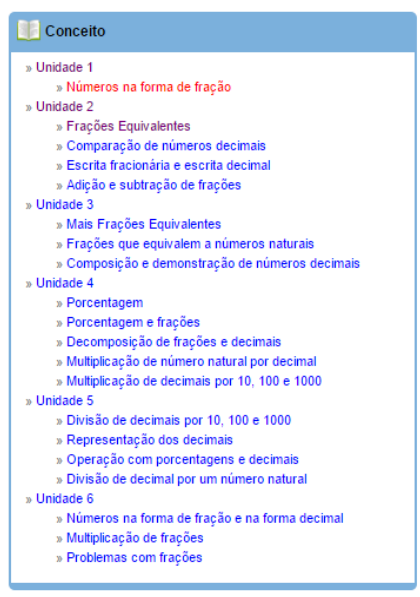

(b)

Figura 3 - (a) Visualização de um OA e (b) Menu com as UAs

O Quadro 1 apresenta o progresso geral das participantes e o Quadro 2 o desempenho.

\section{Quadro 1 - Progresso geral das participantes}

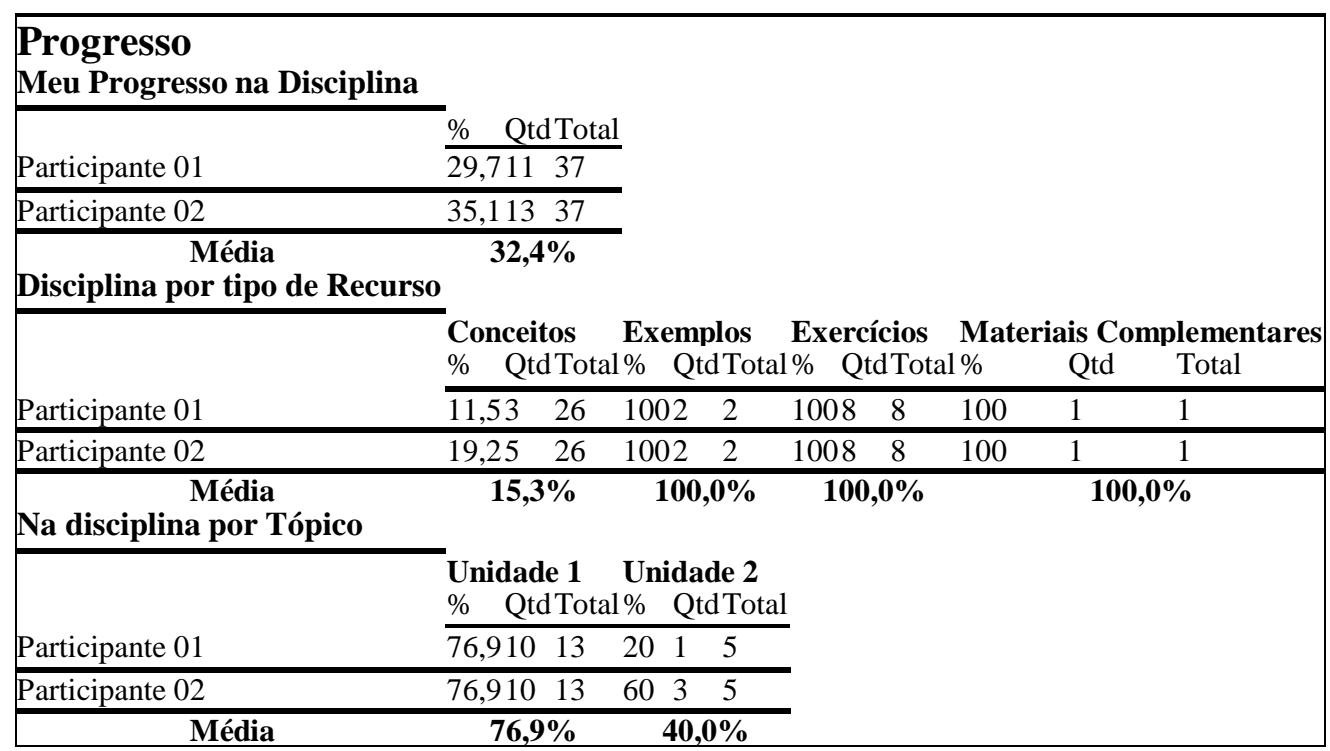




\section{Quadro 2 - Desempenho geral das participantes}

\begin{tabular}{|c|c|c|}
\hline $\begin{array}{l}\text { Desempenho } \\
\text { Meu desempenho nos exercícios }\end{array}$ & & \\
\hline & \% OtdTotal & \\
\hline Participante 01 & $\overline{12,578}$ & Errou Questão 8 \\
\hline Participante 02 & $12,57 \quad 8$ & Errou Questão 8 \\
\hline $\begin{array}{c}\text { Média } \\
\text { Meu desempenho na avaliação }\end{array}$ & $12,5 \%$ & \\
\hline Participante 01 & $\begin{array}{l}\frac{\%}{\text { Otd }} \text { lotal } \\
77,77 \quad 9\end{array}$ & Errou Questões 1, 5 e 9 \\
\hline Participante 02 & $88,88 \quad 9$ & Errou Questões 1 e 5 \\
\hline Média & $83,3 \%$ & * Questão 5 anulada \\
\hline
\end{tabular}

Os exercícios e a avaliação estão inseridos no ambiente, sendo apresentados por questões fechadas (por exemplo, questões de múltipla escolha, verdadeiro e falso, etc.) e abertas (discursivas). Quanto a realização dos exercícios, as participantes poderiam se comunicar por meio de ferramentas de comunicação adicionais (Skype, chat, etc.), para resolver as questões. Já para a avaliação esses recursos de comunicação não foram utilizados, e cada participante a realizou de forma individual.

\subsection{Questionário de Satisfação}

Este questionário foi respondido pelas participantes juntamente com o observador que as acompanhou durante a avaliação. As questões destacadas em vermelho não puderam ser avaliadas pelas participantes, pois as participantes não acessaram os exemplos propostos. Além das questões objetivas, as participantes realizaram pontuações sobre a experiência. A Tabela 1 apresenta a pontuação de cada participante sobre a Experiência e a Tabela 2 sobre sua Satisfação.

Tabela 1. Pontuações das Participantes sobre a Experiência

\begin{tabular}{|l|l|l|}
\hline \multirow{2}{*}{ Participante 01 } & Sobre o Conteúdo & Pontos Negativos \\
\cline { 2 - 3 } & Pontos Positivos & $\begin{array}{l}\text { Demora para fazer uma conta e saber } \\
\text { onde começar as atividades }\end{array}$ \\
\hline Participante 02 & Jogo, Exercícios e Explicações & $\begin{array}{l}\text { Não foi possível assistir ao vídeo } \\
\text { (referindo-se aos exemplos) }\end{array}$ \\
\hline & Sobre a Ferramenta & Pontos Negativos \\
\cline { 2 - 3 } & Pontos Positivos & $\begin{array}{l}\text { Na avaliação as questões dos exercícios } \\
\text { foram citadas de novo }\end{array}$ \\
\hline Participante 01 & Explicou bem o que é fração & $\begin{array}{l}\text { Não foi possível assistir ao vídeo } \\
\text { (referindo-se aos exemplos) }\end{array}$ \\
\hline Participante 02 & Jogo (Enigma das Frações) &
\end{tabular}

Tabela 2. Resultados do Questionário de Satisfação

\section{Questionário de Satisfação}

Sobre sua motivação

Me senti motivado a participar durante todo o curso

Gostei do assunto abordado (matemática/frações)

Sobre o conteúdo

\begin{tabular}{cc}
$\begin{array}{c}\text { Participante } \\
\text { 01 }\end{array}$ & $\begin{array}{c}\text { Participante } \\
\text { 02 }\end{array}$ \\
\hline 10 & 7 \\
10 & 10 \\
\hline
\end{tabular}


Achei o conteúdo claro e fácil de entender Achei a quantidade de exercícios suficiente

Achei a quantidade de materiais complementares suficiente

Sobre seus estudos

Vi todo o conteúdo

Resolvi todos os exercícios

Vi todos os exemplos

Vi todos os materiais complementares

Aprendi o que são frações e como representá-las

Sobre o sistema

Achei o sistema fácil de utilizar

Consegui navegar no conteúdo do minicurso sem dificuldade

Consegui acessar os exemplos sem dificuldade

Consegui resolver os exercícios sem dificuldade

Consegui acessar os materiais complementares sem dificuldade

Consegui enviar recados aos meus professores e colegas sem dificuldades

Consegui utilizar o fórum de discussão sem dificuldades

Média

Geral: 9.1

\section{0}

10

10

7

10

7

$10 \quad 10$

$10 \quad 10$

$\begin{array}{ll}7 & 10\end{array}$

$7 \quad 10$

$10 \quad 10$

$\begin{array}{cc}7 & 7 \\ 10 & 10\end{array}$

$10 \quad 10$

$10 \quad 10$

$\begin{array}{ll}7 & 7\end{array}$

9.2

\section{Análise dos Resultados Obtidos}

A avaliação foi realizada por meio de um estudo de caso com duas participantes e teve um caráter qualitativo. Mesmo fazendo uso de métricas objetivas como o Inventário de Estilo de Aprendizagem de Felder, desempenho de progresso analítico e o questionário de satisfação, a observação e a entrevista realizada com as participantes foram os instrumentos mais importantes para a análise dos dados.

Considerando os objetivos pedagógicos definidos neste projeto e o processo de avaliação realizado, foi possível verificar que houve um resultado positivo após a utilização do OA no que se refere a interpretação de diferentes tipos de desenhos e gráficos referentes a frações. Ambas as participantes declaram ter aprendido tais conceitos o que ficou evidenciado tanto nos exercícios como na avaliação.

Com relação aos estilos de aprendizagem estabelecidos como sendo favorecidos pela abordagem adotada, a Participante 01 apresentou um equilíbrio em todas as dimensões, enquanto a Participante 02 mostrou-se moderadamente intuitiva $\mathrm{e}$ sequencial e fortemente visual. Considerando a percepção de cada uma quanto à sua aprendizagem, o resultado do inventário corrobora a proposta do projeto.

Neste estudo de caso não foi possível mensurar a colaboração entre as participantes, entretanto, elas não só buscaram a interação entre si, como a Participante 02 demonstrou claramente a necessidade de mediação (na figura da integrante responsável pela observação) tanto durante a avaliação quanto na entrevista final.

\section{Considerações Finais}

Este projeto viabilizou, na prática, a constatação da importância da utilização de uma metodologia para que DI de OAs alcancem seus objetivos. A metodologia adotada, contemplou desde a etapa de análise e concepção da ideia até sua avaliação final onde foi possível identificar pontos a melhorar e aprimorar. 
Também foi possível perceber o quão importante é a participação de todos os envolvidos de forma efetiva para que o objeto de aprendizagem cumpra seu papel pedagógico por meio da tecnologia. Assim sendo, não há protagonistas neste processo, mas sim, profissionais trabalhando em conjunto onde cada um dos envolvidos contribui com seu expertise, técnicas e habilidades para que o resultado final se converta em aprendizagem daqueles que farão uso da ferramenta.

A etapa de avaliação mostrou-se extremamente válida para que fosse possível observar aspectos importantes como a usabilidade, assimilação de conteúdos e, principalmente, aspectos a serem aprimorados ou mesmo descartados. Acredita-se que esta primeira avaliação sirva como input para a continuidade dos trabalhos considerando uma amostragem maior de forma que a mesma possa assumir um caráter quantitativo, contribuindo ainda mais para o aprimoramento da solução.

\section{Referências}

CASTRO, A. MENEZES, C. Aprendizagem Colaborativa com suporte computacional. In: Pimentel, MFulks, H. (Org.). Sistemas Colaborativos. Rio de Janeiro: CampusElsevier, 2011, p 135-153.

FELDER, R. M., SILVERMAN, L. K. Learning and Teaching Styles in Engineering Education, Journal of Engineering Education, v.78, n.7, p. 674-681, 1998.

FELDER, R. M., BRENT, R. Understanding Student Differences. Journal of Engineering Education, v. 94, n. 1, p. 57-72, 2005.

FILATRO, A. Design Instrucional na prática. São Paulo: Pearson Education do Brasil, 2008.

GASPARINI, I Interface Adaptativa no ambiente AdaptWeb: navegação e apresentação adaptativa baseada no modelo do usuário. Diss. Universidade Federal Do Rio Grande Do Sul. 2003.

ILSQ - Index of Learning Styles Questionnaire, Disponível em: https://www.engr.ncsu.edu/learningstyles/ilsweb.html. Acesso em Outubro de 2015.

LA TAILlE, Y.; OLIVEIRA, M. K.; DANTAS, H. Piaget, Vygotsky, Wallon teorias psicogenéticas em discussão. São Paulo: Summus. 1992.

SANTOS, A. A. A. DOS; MOGNON, J. F. (2010). Estilos de aprendizagem em estudantes universitários. Boletim de psicologia, v. 60, n. 133, p. 229-241, 2010.

VYGOTSKY, L. S. A formação social da mente: o desenvolvimento dos processos psicológicos superiores.7. ed. São Paulo: Martins Fontes, 2007.

WILEY, D. Learning objects need instructional design theory. The ASTD e-Learning handbook, 2002. 